\title{
Del Apego Adulto a la Infidelidad Sexual: Un Análisis de Mediación Múltiple
}

\section{From Adult Attachment to Sexual Infidelity: A Multiple Mediation Analysis}

\author{
Ana Yngrid Díaz-Pichardo \\ Universidad Autónoma de Santo Domingo \\ Luis Eduardo Garrido \\ Pontificia Universidad Católica Madre y Maestra \\ Cayetano Aranda Torres y Tesifón Parrón-Carreño \\ Universidad de Almería
}

\begin{abstract}
Una gran proporción de las relaciones de pareja sufre problemas de infidelidad, impactándose sustancialmente la calidad de vida de sus miembros, sus familias y la sociedad. A pesar de que numerosos estudios han encontrado una relación directa entre el apego inseguro y la infidelidad, es menos lo que se sabe sobre las variables que podrían estar mediando dicha relación. Bajo este contexto, el objetivo del presente estudio fue esclarecer la relación entre el apego adulto y la infidelidad sexual, considerando 3 posibles mediadores: la satisfacción con la relación de pareja y la percepción de las consecuencias positivas y negativas de la infidelidad. Se obtuvo una muestra por conveniencia de 403 estudiantes de una universidad pública de Santo Domingo, República Dominicana, con edades comprendidas entre los 18 y 69 años $(M=28,05, D E=8,69)$. El apego adulto se midió con el instrumento Experiencias en Relaciones Cercanas, la satisfacción con la relación de pareja, con el Índice de Satisfacción de Parejas y la infidelidad sexual y las consecuencias positivas y negativas de la infidelidad, mediante el Inventario Multidimensional de Infidelidad, Los resultados indicaron que tanto el apego evitativo como ansioso correlacionaron directamente con la infidelidad sexual y que esta relación estuvo mediada en gran medida, de acuerdo a un modelo de análisis de rutas con bootstraping, por la satisfacción con la relación de pareja y las consecuencias positivas de la infidelidad. Estos resultados son examinados a partir de la teoría del apego y se proponen líneas futuras de investigación.
\end{abstract}

Palabras clave: apego, infidelidad, satisfacción en la relación, relación de pareja, sexualidad

\begin{abstract}
A large proportion of couples experience infidelity issues, which substantially impact the quality of life of its members, their families, and society. Although numerous studies have found a positive association between insecure attachment and infidelity, less is known about the variables that may mediate this link. In this context, the objective of this study was to clarify the association between adult attachment and sexual infidelity, considering 3 possible mediators: participants' satisfaction with their couple relationships and their perception of the positive and negative consequences of infidelity. A convenience sample of 403 university students was obtained at a public university in Santo Domingo, Dominican Republic. Participants' age ranged from 18 to 69 years $(\mathrm{M}=$ 28.05, SD = 8.69). Adult attachment was measured with the Experiences in Close Relationships instrument, relationship satisfaction with the Couples Satisfaction Index, and sexual infidelity and the positive and negative consequences of infidelity with the Multidimensional Inventory of Infidelity. Results indicated that both avoidant and anxious attachment were positively correlated with sexual infidelity and that this association was largely mediated -according to a path analysis model with bootstrapping- by relationship satisfaction and a positive perception of the consequences of infidelity. These results are examined upon the basis of attachment theory. Lastly, future lines of research are proposed.
\end{abstract}

Keywords: attachment, infidelity, couples satisfaction, relationship, sexuality

Ana Yngrid Díaz-Pichardo, Instituto de Sexualidad Humana, Escuela de Medicina, Universidad Autónoma de Santo Domingo, República Dominicana; Luis Eduardo Garrido, Vicerrectoría de Investigación e Innovación, Pontificia Universidad Católica Madre y Maestra, Santo Domingo, República Dominicana; Cayetano Aranda Torres, Departamento de Filosofía, Facultad de Humanidades, Universidad de Almería, España; Tesifón Parrón-Carreño, Departamento de Enfermería, Fisioterapia y Medicina, Universidad de Almería, España.

La correspondencia relativa a este artículo debe ser dirigida a Ana Yngrid Díaz-Pichardo, Instituto de Sexualidad Humana, Escuela de Medicina, Universidad Autónoma de Santo Domingo, Aristides Fiallo Cabral, Ciudad Universitaria, Hospital Marion, Santo Domingo 10105, República Dominicana. E-mail: anaydiazp@hotmail.com 
DÍAZ-PICHARDO, GARRIDO, ARANDA TORRES Y PARRÓN-CARREÑO

En las relaciones de pareja la infidelidad ha sido objeto de muchas investigaciones, encontrándose altos niveles de frecuencia en distintas poblaciones (Allen et al., 2005; Zare, 2011). Según Fisher (1992/2007), en las sociedades occidentales más del $50 \%$ de las personas casadas había estado involucrada en una infidelidad. Asimismo, un tercio de los universitarios informaron haber engañado sexual y/o emocionalmente a su pareja y otro grupo similar afirma que su pareja le ha sido infiel (Hall \& Fincham, 2009; López Beltrán, Pérez Barberá, Piquer Barrachina \& Ballester-Arnal, 2017). La prevalencia de la infidelidad es mayor entre las personas que no están casadas, mostrando los jóvenes actitudes más permisivas hacia las implicaciones de la infidelidad (Logue, 2013). En parejas heterosexuales, los hombres tienen mayor probabilidad de participar en una infidelidad que las mujeres (Allen et al, 2005); estas diferencias se asocian a los roles de género, a los procesos de socialización, a las creencias sociales sobre lo propio para cada sexo y a las diferencias culturales y étnicas (Buunk \& Dijkstra, 2015; Parker \& Campbell, 2017). A pesar de esto, la mayoría de las personas casadas espera que su cónyuge tenga relaciones sexuales solo en el matrimonio y supone que su compañero espera exclusividad sexual de ellos (Boekhout, Hendrick \& Hendrick, 2003; Treas \& Giesen, 2000).

Ha sido difícil llegar a acuerdos sobre lo que las personas entienden por infidelidad. Al hablar de infidelidad, es necesario tomar en cuenta aspectos del ser humano, como lo genético, neurológico, cultural y psicológico, siendo estos algunos de los muchos indicadores que marcan la diferencia (Hatfield \& Rapson, 2006). Asimismo, se incluye la edad, el estado civil y la nacionalidad, entre otros, los que siguen siendo objeto de estudio (Logue, 2013). En el contexto de este trabajo, la infidelidad se definirá, a partir de Afifi, Falato y Weiner (2001), como una conducta sexual y/o romántica que se presenta fuera de la relación convenida entre miembros de la pareja, ya sea esta una relación de noviazgo, matrimonio o de cohabitación, en la cual se tiene la expectativa de exclusividad y formalidad.

La infidelidad tiene un impacto considerable en la calidad de vida individual, familiar y de la sociedad (Giraldo Hurtado \& Garcés, 2013; Lewandowski \& Ackerman, 2006; Rivera Aragón, Díaz Loving, Villanueva Orozco \& Montero Santamaria, 2011; Russell, Backer \& McNulty, 2013). Debido a esto, numerosas investigaciones han indagado sobre su posible relación con diversas variables psicológicas, siendo algunas de las más relevantes la satisfacción con la relación de pareja (SRP), el estilo de apego adulto y las actitudes hacia la infidelidad. Así, se ha encontrado que son más infieles aquellas personas que reportan una menor SRP (Allen, Rhoades, Stanley, Loew \& Markman, 2012; Mark, Janssen \& Milhausen, 2011; Martins et al., 2016; McDaniel, Drouin \& Cravens, 2017), tienen un apego inseguro (DeWall et al., 2011; McDaniel et al., 2017; Parker \& Campbell, 2017; Russell et al., 2013) y muestran actitudes más positivas hacia la infidelidad (DeWall et al., 2011; Martins et al., 2016; Romero-Palencia, Cruz del Castillo \& Díaz-Loving, 2008). A pesar de estos hallazgos, existe una carencia de estudios sobre infidelidad que consideren estas variables de manera conjunta y que permitan, por lo tanto, indagar sobre posibles roles mediadores de algunas de ellas.

Estudiar de manera conjunta los efectos de múltiples variables mediadoras tiene importantes ventajas, entre las que están (Icard, Jemmott III, Teitelman, O'Leary \& Heeren, 2014; Preacher \& Hayes, 2008): (a) un modelo de mediación múltiple permite determinar hasta qué punto una variable específica media el efecto entre una variable independiente y una dependiente, condicional a (o controlando por) la presencia de otros mediadores; (b) si se suman los efectos indirectos de varios modelos de mediación simples para obtener el efecto total indirecto de múltiples mediadores, se puede producir sesgo, debido a que los mediadores típicamente correlacionan entre sí; (c) cuando se consideran varios mediadores putativos en un modelo de mediación múltiple, se reduce la probabilidad de sesgo en los parámetros producto de variables omitidas y (d) los modelos de mediación múltiple permiten determinar la magnitud relativa de los efectos indirectos específicos asociados con todos los mediadores. Tomando en cuenta lo expuesto, el objetivo del presente estudio fue examinar la relación entre el apego adulto y la infidelidad sexual, con las variables de SRP y actitudes hacia la infidelidad como posibles mediadoras de dicha relación.

\section{El Apego Adulto, la Satisfacción con la Relación y las Actitudes hacia la Infidelidad}

Según la teoría del apego formulada por John Bowlby (1969/1998), existe una necesidad humana universal de formar vínculos afectivos estrechos hacia los cuales recurrir en momentos de sufrimiento o estrés. A nivel general, el sistema de apego constituye un patrón de emociones, cogniciones y comportamientos que organizan la actividad humana a través del ciclo vital (DeWall et al., 2011). El sistema de apego tiene para Bowlby (1969/1998) bases biológicas. Plantea que los comportamientos de 
apego hacen posible la aproximación del niño a la figura cuidadora y protectora (generalmente la madre). Algunos de esos comportamientos, como sonreír y vocalizar, anuncian al adulto el interés del niño en la interacción, promoviendo el acercamiento. Otros comportamientos, como el llanto, también invitan al acercamiento, pero con el fin de detenerlos.

Aunque inicialmente esta teoría se focalizó en el vínculo formado en las relaciones tempranas, luego fue aplicada al amor de pareja, sosteniendo que el comportamiento del adulto en relaciones cercanas está modelado por representaciones mentales cuyos orígenes se encuentran en las relaciones del niño con sus cuidadores primarios (Hazan \& Shaver, 1987). Bowlby (1969/1998) considera que los sistemas de apego infantiles son similares en su naturaleza a los que más tarde entran en juego en las relaciones amorosas y señala pocas diferencias entre las relaciones cercanas, sean estas entre padres e hijos o entre pares. Los elementos del comportamiento del apego en la vida adulta son similares a los observados en la infancia. Un adulto muestra deseo hacia la proximidad de figuras de apego en situaciones de malestar, siente bienestar ante la presencia de esa figura y ansiedad si esta es inaccesible. La angustia es esperable ante la pérdida de una figura de apego. Dependiendo de cómo fue el apego con los cuidadores de infancia, se crea una impresión en el transcurso de la vida sobre cómo confiar en otro adulto en el contexto de la relación de pareja. Las personas que tuvieron padres cálidos, amorosos, confiables y que eran una fuente de apoyo emocional, tienden a formar un apego seguro (Hazan \& Shaver, 1994). Estas forman relaciones íntimas, duraderas y profundas, caracterizadas por la confianza y el deseo de intimidad, mientras que las personas cuyos padres eran distantes o fríos, tienden a tener un apego inseguro en la vida adulta (Hazan \& Shaver, 1994).

Bowlby (1969/1998) señaló que las personas varían en sus tendencias hacia el apego evitativo (EVI) y el apego ansioso (ANS). Las personas que puntúan alto en EVI tienden a sentirse incómodos con la cercanía e intimidad, lo que les lleva a hacer frente a estos sentimientos alejándose de su pareja (Fraley \& Shaver, 2000; Mikulincer \& Shaver, 2003, 2007). Estas personas son suspicaces, escépticas y retraídas, se sienten incómodas intimando con otros, encuentran difícil confiar y depender, valoran la autosuficiencia, tienen miedo a ser rechazadas, presentan insatisfacción en las relaciones de pareja y dan más importancia a su realización personal (Davis, Shaver \& Vernon, 2003; Loubat, Ponce \& Salas, 2007). En cambio, las personas que obtienen alta puntuación en el ANS tienden a experimentar ambivalencia, indicada por la coexistencia del deseo de cercanía y evitación del rechazo (Campbell, Simpson, Boldry \& Kashy, 2005), dudan que puedan ser queridos y tienen una visión idealizada de los otros, aunque se decepcionan fácilmente de las personas. Buscan relaciones cercanas para elevar su autoestima, son dependientes, sobreinterpretan las emociones y conductas de los demás, son hipervigilantes y tienen altos niveles de angustia y miedo constante de ser abandonados, por lo que son controladores (Davis et al., 2003; Loubat et al., 2007). Considerando estos aspectos, Fraley y Shaver (2000) plantean que la dimensión de evitación mide las estrategias de comportamiento que las personas usan para regular sus necesidades de apego y la dimensión de ansiedad mide los procesos afectivos y atributos involucrados en monitorear y evaluar eventos para detectar signos de amenazas.

Cuando dos individuos deciden formalizar su relación y ser pareja, pasan a integrar una nueva unidad social. Este sistema es más que la suma de dos personas que se relacionan, esta unión da lugar a una nueva realidad cualitativamente distinta (Elsner, Montero, Reyes \& Zegers, 2000). Sea como fuere la historia individual de cada uno, los estilos de apego que hayan tenido en su relación primaria y el tipo de relación que hayan mantenido anteriormente, el nuevo sistema comienza a establecer pautas de interacción, cuyo propósito puede complementar o contrariar los objetivos individuales y de la pareja (Cole, 1997/2002). La fragilidad y las carencias afectivas del vínculo emocional de la pareja son dos de los principales motivos que llevan a la infidelidad, independientemente del género (López Beltrán et al., 2017; Mark et al., 2011; Weiser, Lalasz, Weigel \& Evans, 2014). Asimismo, los jóvenes adultos atribuyen su conducta de infidelidad principalmente a sus relaciones primarias y/o a que sus parejas no satisfacen sus necesidades de interdependencia (Norona, Olmstead \& Welsh, 2018), y los comportamientos de infidelidad son más probables en aquellas personas que tienen actitudes y conceptualizaciones más positivas hacia la misma (Romero-Palencia et al., 2008; Ziv, Lubin \& Asher, 2018).

Sentirse cerca y comprometido con la pareja inhibe el interés de las personas en buscar alternativas y participar en la infidelidad (DeWall et al., 2011). Kobak y Hazan (1991) habían anticipado que el apego seguro de los cónyuges estaría vinculado a la regulación emocional constructiva durante dos tipos de interacción conyugal: la resolución de problemas y la conducta de confiar. De igual modo, se ha comprobado que el apego está vinculado de manera teóricamente predecible a la satisfacción marital y sexual (Butzer \& 
Campbell, 2008; Medina, Rivera \& Aguasvivas, 2016). Las personas con apego inseguro advierten menores niveles de satisfacción marital ( $\mathrm{Li} \&$ Chan, 2012), pudiendo esperarse que quienes presenten EVI o ANS, al elegir a sus parejas, experimenten un mayor nivel de insatisfacción marital en el marco de sus relaciones conyugales. La ocurrencia de esta asociación se debe a que las personas con altos niveles de evitación establecen relaciones superficiales carentes de vinculación cercana, expresión afectiva y sostenimiento de la intimidad. De igual manera, los altos niveles de ansiedad tienden a generar ambivalencia, manifestada por el deseo de cercanía y el deseo de evitar el rechazo (DeWall et al., 2011; Mikulincer \& Shaver, 2003).

La literatura previa sugiere que existe un proceso causal que lleva a las personas con apego inseguro a ser más infieles y que este proceso está afectado por la SRP y las actitudes hacia la infidelidad. Por un lado, estaría el proceso que lleva del apego inseguro a una menor SRP y posteriormente a la infidelidad. En este proceso el EVI afecta la SRP, debido a que las personas rehúyen de la dependencia, intimidad y compromiso en sus relaciones, mientras que aquellos con ANS tienen menor satisfacción, debido a que son demasiado sensibles a las señales de rechazo en las relaciones y adoptan actitudes de hipervigilancia (Li \& Chan, 2012). A su vez, estudios longitudinales han encontrado que la SRP puede ser tanto una causa como una consecuencia de la infidelidad (Fincham \& May, 2017; Previti \& Amato, 2004), siendo, además, señalada como una razón principal cuando se les pregunta directamente a las personas sobre la razón de su infidelidad (Martins et al., 2016). Por otro lado, se tendría el proceso que lleva del apego inseguro a actitudes más positivas hacia la infidelidad y luego a la conducta infiel. Aquí, las personas con EVI, debido a que se sienten crónicamente incómodas con la intimidad y cercanía emocional, tienden a estar más interesadas en alternativas a su pareja y a tener actitudes más positivas hacia la infidelidad (DeWall et al., 2011). En el caso de las personas con ANS, la necesidad de tener mayor intimidad puede hacer que estas personas tengan actitudes más positivas hacia la infidelidad, ya que las relaciones extradiádicas serían una forma de satisfacer esta necesidad (Russell et al., 2013). A su vez, tener actitudes más positivas hacia la infidelidad hace que las personas sean más infieles, un resultado esperable, dada la literatura sobre las actitudes como predictores del comportamiento (DeWall et al., 2011; Martins et al., 2016).

\section{El Presente Estudio}

El objetivo del presente estudio fue esclarecer la relación entre el apego adulto (EVI y ANS) y la infidelidad sexual, considerando tres posibles mediadores: la SRP y la percepción de las consecuencias positivas (CPI) y negativas (CNI) de la infidelidad. El estudio se enfocó en la infidelidad sexual, debido a que tanto mujeres como hombres presentan una mayor consistencia en la forma que la definen, a diferencia de la infidelidad emocional, que constituye un concepto más ambiguo y complejo (Guitar et al., 2017). Se esperaba que ambos tipos de apego se relacionaran directamente con la infidelidad sexual, aunque de manera mayor el EVI (DeWall et al., 2011). Por el contrario, se esperaba que tanto el EVI como el ANS se relacionaran de manera inversa con la SRP, aunque con mayor fuerza el EVI (Li \& Chan, 2012; Medina et al., 2016). A su vez, se esperaba que la SRP se relacionara de manera inversa con la infidelidad sexual (DeWall et al., 2011; Previti \& Amato, 2004). Adicionalmente, se previó una relación directa entre la infidelidad sexual y CPI y una relación inversa entre infidelidad sexual y CNI (Romero-Palencia et al., 2008; Ziv et al., 2018). Finalmente, se esperaba que SRP, CPI y CNI mediaran la relación entre el apego y la infidelidad sexual.

\section{Método}

\section{Participantes}

La muestra inicial estuvo compuesta por 435 estudiantes seleccionados por conveniencia de una gran universidad pública localizada en Santo Domingo, República Dominicana. De estos, 16 fueron eliminados por haber completado la batería sin tener una relación de pareja. Seis casos fueron eliminados porque seleccionaron la opción 2 en el ítem de control sobre el tipo de relación de pareja (tenían una relación abierta) y otros seis fueron eliminados por responder de manera correcta a menos del $80 \%$ de los ítems de control atencional. Finalmente, y siguiendo las recomendaciones de Hair Jr., Black, Babin y Anderson (2010), cuatro casos se eliminaron porque dejaron sin responder a más del 50\% de los ítems de alguno de los instrumentos. La muestra final, por lo tanto, quedó compuesta por 403 estudiantes. 
Los 403 estudiantes universitarios que conformaron la muestra final tenían edades comprendidas entre los 18 y los 69 años $(M=28,05, D E=8,69)$. Asimismo, la muestra final estuvo compuesta por 270 mujeres $(67,0 \%)$ y 133 hombres $(33,0 \%)$, de los cuales la mayoría eran solteros $(50,6 \%)$, no estaban trabajando (46,2\%), su titulación máxima era de bachiller (50,6\%), estaban principalmente cursando titulaciones en las áreas de ciencias de la salud $(22,1 \%)$, ingeniería y arquitectura $(20,6 \%)$ y humanidades $(17,6 \%)$, de religión católica $(40,7 \%)$ y de orientación sexual heterosexual (91,6\%) (Tabla 1). Adicionalmente, los participantes tenían una media de 0,70 hijos $(D E=1,25), 3,88$ hermanos $(D E=2,55)$ y percibían un promedio de 21.920 pesos dominicanos al mes $(D E=27.980)$ (Tabla 2). En términos de la relación de pareja (Tabla 2), la duración de esta al momento de la recogida de datos era entre 6 meses y 48 años 2 meses $(M=5,66, D E=$ $7,06)$ y un $43,7 \%$ respondió que durante su relación actual había tenido contacto sexual en secreto con una persona distinta a su pareja (Tabla 1). En las Tablas 1 y 2, además de mostrar los estadísticos descriptivos para las variables sociodemográficas categóricas y continuas, se muestran las comparaciones por género para dichas variables.

Tabla 1

Estadísticos Descriptivos para las Variables Sociodemográficas Categóricas

\begin{tabular}{|c|c|c|c|c|c|c|}
\hline \multirow{2}{*}{ Variable/Categoría } & \multirow{2}{*}{$\begin{array}{c}\begin{array}{c}\text { Total } \\
(n=403)\end{array} \\
N(\%)\end{array}$} & \multirow{2}{*}{$\begin{array}{c}\begin{array}{c}\text { Mujeres } \\
(n=270)\end{array} \\
N(\%)\end{array}$} & \multirow{2}{*}{$\begin{array}{c}\begin{array}{c}\text { Hombres } \\
(n=133)\end{array} \\
N(\%)\end{array}$} & \multicolumn{3}{|c|}{ Prueba $\chi^{24,5}$} \\
\hline & & & & $\chi^{2}$ & $g l$ & $p$ \\
\hline Estado civil & & & & 14,81 & 2 & 0,001 \\
\hline Soltero/a & $204(50,6)$ & $\underline{121(44,8)}$ & $\underline{83(62,4)}$ & & & \\
\hline Casado/a & $105(26,1)$ & $\underline{85(31,5)}$ & $\underline{20(15,0)}$ & & & \\
\hline Cohabitando & $89(22,1)$ & $\overline{61(22,6)}$ & $\overline{28(21,1)}$ & & & \\
\hline Otro $^{1}$ & $5(1,2)$ & $3(1,1)$ & $2(1,5)$ & & & \\
\hline Estatus laboral & & & & 4,90 & 2 & 0,086 \\
\hline No trabaja & $186(46,2)$ & $135(50,0)$ & $51(38,3)$ & & & \\
\hline Tiempo parcial & $107(26,6)$ & $66(24,4)$ & $41(30,8)$ & & & \\
\hline Tiempo completo & $110(27,3)$ & $69(25,6)$ & $41(30,8)$ & & & \\
\hline Nivel educativo & & & & 13,59 & 3 & 0,004 \\
\hline Bachiller & $204(50,6)$ & $134(49,6)$ & $70(52,6)$ & & & \\
\hline Técnico & $72(17,9)$ & $\underline{38(14,1)}$ & $\underline{34(25,6)}$ & & & \\
\hline Grado/licenciatura & $86(21,3)$ & $64(23,7)$ & $22(16,5)$ & & & \\
\hline Posgrado/máster/doctorado & $41(10,2)$ & $\underline{34(12,6)}$ & $\underline{7(3,8)}$ & & & \\
\hline Área de estudio & & & & 126,06 & 8 & $<0,001$ \\
\hline Agronómica y veterinaria & $5(1,2)$ & $\underline{0(0,0)}$ & $\underline{5(3,8)}$ & & & \\
\hline Artes & $19(4,7)$ & $\overline{8(3,0)}$ & $11(8,3)$ & & & \\
\hline Ciencias & $12(3,0)$ & $\overline{10(3,7)}$ & $2(1,5)$ & & & \\
\hline Ciencias de la salud & $89(22,1)$ & $73(27,0)$ & $16(12,0)$ & & & \\
\hline Económicas y sociales & $31(7,7)$ & $21(7,8)$ & $10(7,5)$ & & & \\
\hline Educación & $30(7,4)$ & $27(10,0)$ & $\underline{3(2,3)}$ & & & \\
\hline Humanidades & $71(17,6)$ & $\underline{62(23,0)}$ & $\underline{9(6,8)}$ & & & \\
\hline Ingeniería y arquitectura & $83(20,6)$ & $16(5,9)$ & $6 \overline{(50,4)}$ & & & \\
\hline Jurídicas y políticas & $24(6,0)$ & $\overline{17(6,3)}$ & $7(5,3)$ & & & \\
\hline Sin especificar & $39(9,7)$ & $36(13,3)$ & $3(2,3)$ & & & \\
\hline Religión & & & & 10,77 & 4 & 0,029 \\
\hline Adventista & $11(2,7)$ & $7(2,6)$ & $4(3,0)$ & & & \\
\hline Católica & $164(40,7)$ & $117(43,3)$ & $47(35,3)$ & & & \\
\hline Cristiana & $58(14,4)$ & $38(14,1)$ & $20(15,0)$ & & & \\
\hline Evangélica & $60(14,9)$ & $46(17,0)$ & $14(10,5)$ & & & \\
\hline Ninguna & $102(25,3)$ & $\underline{56(20,7)}$ & $46(34,6)$ & & & \\
\hline Otra $^{2}$ & $8(2,0)$ & $6(2,2)$ & $2(1,5)$ & & & \\
\hline
\end{tabular}


Tabla 1 (conclusión)

Estadísticos Descriptivos para las Variables Sociodemográficas Categóricas

\begin{tabular}{|c|c|c|c|c|c|c|}
\hline \multirow{2}{*}{ Variable/Categoría } & $\begin{array}{c}\text { Total } \\
(n=403)\end{array}$ & $\begin{array}{l}\text { Mujeres } \\
(n=270)\end{array}$ & $\begin{array}{l}\text { Hombres } \\
(n=133)\end{array}$ & \multicolumn{3}{|c|}{ Prueba $\chi^{24,5}$} \\
\hline & $N(\%)$ & $N(\%)$ & $N(\%)$ & $\chi^{2}$ & $g l$ & $p$ \\
\hline Orientación sexual & & & & 0,01 & 1 & 0,932 \\
\hline Homosexual & $19(4,7)$ & $13(4,8)$ & $6(4,5)$ & & & \\
\hline Heterosexual & $369(91,6)$ & $249(92,2)$ & $120(90,2)$ & & & \\
\hline Otra $^{3}$ & $14(3,5)$ & $7(2,6)$ & $7(5,3)$ & & & \\
\hline Sin respuesta & $1(0,2)$ & $1(0,4)$ & $0(0,0)$ & & & \\
\hline Infiel & & & & 38,14 & 1 & $<0,001$ \\
\hline No & $227(56,3)$ & $\underline{181(67,0)}$ & $\underline{46(34,6)}$ & & & \\
\hline Sí & $176(43,7)$ & $\underline{89(33,0)}$ & $\underline{87(65,4)}$ & & & \\
\hline
\end{tabular}

Notas.

1Incluye Divorciado $(N=4)$ y Viudo/a $(N=1)$.

${ }_{2}^{2}$ Incluye Budismo $(N=1)$, Gnosticismo $(N=1)$, Islam $(N=1)$, Mormonismo $(N=1)$ y Testigo de Jehová $(N=4)$.

${ }^{3}$ Incluye Bisexual $(N=6)$ y Asexual $(N=8)$.

${ }^{4}$ Las categorías Otro, Otra y Sin respuesta fueron excluidas de las pruebas de independencia $\chi^{2}$.

${ }^{5}$ Los valores de las casillas con residuos tipificados corregidos significativos $(p<0,05)$ aparecen subrayados.

Tabla 2

Estadisticos Descriptivos para las Variables Sociodemográficas Continuas y las Escalas

\begin{tabular}{|c|c|c|c|c|c|c|c|c|c|c|}
\hline \multirow{2}{*}{ Variable/escala } & \multicolumn{2}{|c|}{$\begin{array}{c}\text { Total } \\
(n=403)\end{array}$} & \multicolumn{2}{|c|}{$\begin{array}{l}\text { Mujeres } \\
(n=270)\end{array}$} & \multicolumn{2}{|c|}{$\begin{array}{l}\text { Hombres } \\
(n=133)\end{array}$} & \multicolumn{4}{|c|}{ Prueba T de Welch } \\
\hline & $M$ & $D E$ & $M$ & $D E$ & $M$ & $D E$ & $t$ & $g l$ & $p$ & $d$ \\
\hline \multicolumn{11}{|l|}{ Variable } \\
\hline Edad & 28,05 & 8,69 & 27,72 & 8,06 & 28,72 & 9,84 & $-1,02$ & 221,6 & 0,311 & 0,12 \\
\hline Hijos & 0,70 & 1,25 & 0,82 & 1,31 & 0,46 & 1,07 & 2,94 & 312,8 & 0,003 & 0,29 \\
\hline Hermanos & 3,88 & 2,55 & 3,94 & 2,54 & 3,75 & 2,57 & 0,71 & 260,1 & 0,478 & 0,08 \\
\hline Ingresos $^{1}$ & 21,92 & 27,98 & 21,22 & 26,42 & 23,34 & 30,97 & $-0,68$ & 229,2 & 0,499 & 0,08 \\
\hline DurRel $^{2}$ & 5,66 & 7,06 & 6,10 & 6,92 & 4,76 & 7,29 & 1,76 & 251,0 & 0,081 & 0,19 \\
\hline \multicolumn{11}{|l|}{ Escala } \\
\hline EVI & 1,95 & 0,48 & 1,95 & 0,50 & 1,93 & 0,44 & 0,40 & 295,7 & 0,693 & 0,04 \\
\hline ANS & 2,35 & 0,53 & 2,38 & 0,52 & 2,30 & 0,54 & 1,30 & 253,9 & 0,196 & 0,14 \\
\hline SRP & 4,46 & 1,11 & 4,50 & 1,14 & 4,38 & 1,05 & 1,05 & 283,1 & 0,296 & 0,11 \\
\hline CNI & 4,28 & 0,77 & 4,32 & 0,77 & 4,19 & 0,76 & 1,59 & 266,9 & 0,114 & 0,17 \\
\hline CPI & 1,90 & 0,84 & 1,78 & 0,78 & 2,15 & 0,91 & $-4,01$ & 229,1 & $<0,001$ & 0,46 \\
\hline IS & 1,55 & 0,76 & 1,35 & 0,61 & 1,95 & 0,87 & $-7,13$ & 198,9 & $<0,001$ & 0,89 \\
\hline
\end{tabular}

Notas. $n=$ tamaño muestral; $M=$ media; $D E=$ desviación estándar; $g l=$ grados de libertad; $d=d$ de Cohen; EVI $=$ Apego evitativo; ANS = Apego ansioso; SRP = Satisfacción con la relación de pareja; $\mathrm{CNI}=$ Consecuencias negativas de la infidelidad; $\mathrm{CPI}=$ Consecuencias positivas de la infidelidad; IS = Infidelidad sexual.

${ }^{1}$ Miles de pesos dominicanos.

${ }^{2}$ DurRel $=$ Duración de la relación de pareja en años.

\section{Instrumentos}

Experiences in Close Relationships (ECR; Brennan, Clark \& Shaver, 1998). El apego adulto se midió a partir del ECR, con la versión adaptada al español y reducida a 32 ítems por Alonso-Arbiol, Balluerka y Shaver (2007). El ECR reducido mide el apego adulto a partir de dos escalas, una de EVI, con 17 ítems (e.g., Prefiero no mostrar a mi pareja cómo me siento por dentro), y otra de ANS, con 15 ítems (e.g., Me preocupa que me abandonen). Alonso-Arbiol et al. (2007) reportan consistencias internas altas para las 
puntuaciones de ambas escalas, con coeficientes alfa de Cronbach de 0,86 y 0,83 para las escalas de EVI y ANS, respectivamente. Asimismo, la escala de EVI obtuvo una confiabilidad test-retest de 0,69 para un periodo de seis semanas, mientras que la de ANS alcanzó una de 0,75. El ECR se aplicó además en República Dominicana en el estudio de Medina et al. (2016), obteniéndose una consistencia interna, evaluada por alfa de Cronbach, de 0,81 para ambas escalas de apego. Desde su creación en el 1998 el ECR ha sido utilizado en cientos de estudios en diversas culturas los cuales proporcionan una amplia evidencia de su validez, incluyendo la robustez de su estructura factorial y la validez de criterio de sus escalas (Alonso-Arbiol et al., 2007). Los ítems del ECR se respondieron a través de una escala de cuatro puntos, con las opciones de muy en desacuerdo (1), en desacuerdo (2), de acuerdo (3) y muy de acuerdo (4). Se optó por una escala de acuerdo-desacuerdo de cuatro puntos (en vez de la original de siete), puesto que las escalas de siete o más opciones pueden deteriorar la calidad de las respuestas, debido a una mayor varianza relacionada a estilos de respuesta (Revilla, Saris \& Krosnick, 2014) y a que solo son recomendables para poblaciones caracterizadas por altos niveles de habilidad cognitiva, destreza verbal y experiencia con cuestionarios (Weijters, Cabooter \& Schillewaert, 2010). Por otro lado, los ítems inversos del ECR reducido, nueve en total, fueron recodificados antes de calcular las puntuaciones en las escalas.

Couples Satisfaction Index en su versión de 16 ítems (CSI-16; Funk \& Rogge, 2007). La SRP se evaluó mediante el CSI-16, el cual fue traducido y adaptado al español para el presente estudio. Tiene una estructura unidimensional y sus autores reportaron una alta consistencia interna, con un a de Cronbach de 0,98. El CSI fue validado originalmente utilizando la teoría de respuesta al ítem, logrando, así, que los ítems retenidos fueran informativos a lo largo del continuo del rasgo (Funk \& Rogge, 2007). Adicionalmente, las puntuaciones del CSI mostraron correlaciones altas con las de otros instrumentos de satisfacción con la relación de pareja y pudieron discriminar adecuadamente entre relaciones de pareja con buen ajuste y relaciones caracterizadas por angustia/estrés (Funk \& Rogge, 2007). Los ítems del CSI-16 (e.g., Tengo una relación cálida y segura con mi pareja) se responden a través de distintas escalas de respuesta (ver Funk \& Rogge, 2007): el ítem 1 cuenta con siete opciones, desde extremadamente infeliz (1) a perfecta (7); el ítem 2 con seis opciones, desde todo el tiempo (1) hasta nunca (6); los ítems 3 a 6 tienen seis opciones, desde para nada verdadero (1) hasta completamente verdadero (6); los ítems 7 a 10 con seis opciones, desde para nada (1) hasta completamente (6) y los ítems 11 al 16 con seis opciones sin etiquetas para indicar entre pares de adjetivos cuál representa mejor la relación de pareja del respondiente. Los cinco ítems inversos del CSI-16 fueron recodificados.

La traducción y adaptación al español del instrumento CSI-16 se llevó a cabo utilizando la técnica paralelo-ciego (Behling \& Law, 2000). Dos traductores bilingües tradujeron los ítems desde la fuente (inglés) a la lengua de destino (español) de manera individual. Luego, ellos, en conjunto con los investigadores y un tercer traductor bilingüe, compararon las dos traducciones, comentaron las diferencias y colaboraron en la creación de una versión final.

Inventario Multidimensional de Infidelidad (IMIN; Romero Palencia, Rivera Aragón \& Díaz Loving, 2007). Las variables de infidelidad sexual y consecuencias de la infidelidad se midieron con el IMIN. La escala de infidelidad sexual del IMIN cuenta con 21 ítems (e.g., He tenido sexo con otra/s persona/s además de mi pareja) y sus autores reportan una consistencia interna a de Cronbach de 0,97. Las consecuencias de la infidelidad se evalúan en el IMIN a partir de dos escalas, una para las CNI, con siete ítems (e.g., La infidelidad destruye las relaciones de pareja), y un a reportado de 0,91, y otra para las CPI (e.g., El tener otra pareja ayuda a soportar los problemas de las relaciones de pareja), la cual se compone de seis ítems y un a de 0,76 en el estudio de validación. Asimismo, los autores mostraron evidencias de la validez de constructo de las escalas del IMIN, a partir de análisis factoriales, en los cuales se obtuvieron agrupaciones de los ítems congruentes con la teoría y cargas factoriales primarias altas. Los ítems de consecuencias de la infidelidad se respondieron a partir de una escala de acuerdo de cinco puntos, con las opciones totalmente en desacuerdo (1), en desacuerdo (2), neutral (3), de acuerdo (4) y totalmente de acuerdo (5). Los ítems de la escala de infidelidad sexual, por su parte, se respondieron a partir de una escala de cinco opciones, que fueron nunca (1), rara vez (2), a veces (3), muy a menudo (4) y siempre (5).

Control atencional. Siguiendo las recomendaciones sobre el control de la inatención (e.g., DeSimone, Harms \& DeSimone, 2015; Maniaci \& Rogge, 2014; Meade \& Craig, 2012), se insertaron cinco ítems dirigidos a lo largo de la batería. Los ítems dirigidos instruían al participante a no responder a ellos (e.g., 
Por favor, deja esta pregunta vacía) y se codificaban con un cero (incorrecto), si el participante marcaba alguna de las opciones de respuesta, y con un uno si los dejaba en blanco (correcto). La consistencia interna a para las puntuaciones obtenidas con los cinco ítems dirigidos fue de 0,74 .

Control del tipo de relación de pareja Para verificar que los respondientes estaban en relaciones de pareja con expectativa de fidelidad se aplicó un ítem que decía ¿Cómo crees que tu pareja describiría la relación que tienen actualmente?. Esta pregunta tenía dos opciones de respuesta: (1) Tu pareja espera que tú no tengas una relación amorosa con nadie más aparte de él/ella y (2) Tu pareja entiende y acepta que tú tengas relaciones amorosas con otras personas aparte de él/ella.

La claridad de los ítems de los instrumentos fue evaluada a partir de un estudio piloto con 22 estudiantes universitarios. Según los resultados de este, se realizaron modificaciones menores en algunos términos, para facilitar la comprensión de los ítems, y se procedió a preparar la batería final con todos los instrumentos.

\section{Procedimiento}

El presente estudio fue aprobado por el comité de ética de la Universidad Autónoma de Santo Domingo (CEI-FCS 004-2017). La recogida de datos se realizó en las aulas de clases de la universidad en horario docente, previa autorización de los profesores de cada asignatura. A los estudiantes que cumplían los criterios de inclusión - habían cumplido los 18 años y estaban en una relación de pareja de 6 meses o más- se les proporcionó el consentimiento informado, el cual fue leído en voz alta por el equipo de investigadores. A aquellos estudiantes que optaron por participar y firmaron el consentimiento informado se les proporcionó la batería de instrumentos y procedieron a responderla de manera anónima. Los estudiantes no recibieron incentivos por participar en el estudio. Cinco estudiantes $(1,1 \%)$ que cumplían con los criterios de inclusión declinaron participar en la investigación.

\section{Análisis Estadístico}

La cantidad de valores perdidos en los instrumentos aplicados fue notablemente baja, con porcentajes de $0,00 \%$ a $0,50 \%$ para los ítems de la batería $(M=0,07 \%, D E=0,13 \%)$. Cuando la cantidad de valores perdidos es pequeña (e.g., < 1 o $2 \%$ ), se considera aceptable hacer una única imputación de los datos (Widaman, 2006). Dada la muy baja cantidad de valores perdidos, los mismos fueron imputados, utilizando el algoritmo EM de esperanza-maximización. El algoritmo EM es considerado como una técnica superior para el manejo de datos perdidos cuando las proporciones de estos no son excesivamente altas, siendo más efectivo que las técnicas tradicionales, como la imputación por la media o por regresión (Fox-Wasylyshyn \& El-Masri, 2005).

Las puntuaciones de las escalas fueron calculadas promediando los ítems que la componían, de forma tal que las mismas mantuvieran el mismo rango de valores que los ítems y fueran así más interpretables.

Para comparar las puntuaciones medias entre mujeres y hombres se utilizó la prueba T de Welch, la cual no asume igualdad de varianza entre los grupos y es recomendada tanto para los casos donde hay heterocedasticidad como para aquellos donde hay homocedasticidad (Delacre, Lakens \& Leys, 2017; Rasch, Kubinger \& Moder, 2011). A pesar de que las puntuaciones de las escalas no tenían una distribución normal, esta prueba resulta apropiada, ya que es robusta al supuesto de normalidad con tamaños suficientemente grandes ( $n \geq 25$; Rasch et al., 2011; Schmider, Ziegler, Danay, Beyer \& Bühner, 2010). Para evaluar el tamaño del efecto se utilizó el estadístico $d$ de Cohen, para el cual valores de 0,20, 0,50 y 0,80 se pueden considerar como indicativos de diferencias pequeñas, medianas y grandes, respectivamente (Cohen, 1992).

Para evaluar la relación lineal entre las puntuaciones de las escalas se utilizó el coeficiente de correlación lineal de Pearson ( $r$ ), siendo sus valores interpretados, según la guía de Cohen (1992): correlaciones de 0,10, 0,30 y 0,50 se consideraron como pequeñas, medianas y grandes, respectivamente.

La consistencia interna de las escalas fue calculada por medio del coeficiente alfa de Cronbach. Para interpretar los valores de consistencia interna se utilizó la guía propuesta por George y Mallery (2003): si $0,90<\alpha \leq 1,00$, se considera excelente; si $0,80<\alpha \leq 0,90$, se considera buena; si $0,70<\alpha \leq 0,80$, se considera aceptable; si $0,60<\alpha \leq 0,70$, se considera cuestionable; si $0,50<\alpha \leq 0,60$, se considera pobre; y si $\alpha<0,50$, la consistencia interna se considera inaceptable. 
Mediante un modelo de rutas se realizó un análisis de mediación múltiple de SRP, CPI y CNI en la relación entre el apego y la infidelidad sexual. La significación estadística y los intervalos confidenciales de los efectos indirectos y directos en los análisis de mediación se llevaron a cabo utilizando la técnica de bootstraping, que ha demostrado un funcionamiento óptimo (Hayes \& Scharkow, 2013). Un total de 50.000 muestras aleatorias con reemplazo fueron generadas a partir de los datos empíricos y para cada una se calculó la magnitud de los efectos; luego, el intervalo confidencial del 95\% se construyó tomando los valores que correspondían a los percentiles 2,5 y 97,5 de la distribución. Efectos indirectos estandarizados de 0,01, 0,09 y 0,25 se consideraron como pequeños, medianos y grandes, respectivamente (Ruiz \& OdriozolaGonzález, 2016). En cuanto a los tamaños muestrales necesarios para detectar efectos indirectos, Fritz y MacKinnon (2007) encontraron que con muestras de 400 se podían detectar la mayoría de los efectos indirectos, inclusive aquellos pequeños. Como suele ser el procedimiento estándar, los errores de las variables mediadoras se correlacionaron. Para el modelo de mediación los indicadores observados fueron las puntuaciones promedio en las escalas y las variables control de edad, sexo, educación y duración de la relación de pareja (Previti \& Amato, 2004). El método de estimación utilizado fue el de máxima verosimilitud, combinado con bootstraping para obtener los intervalos confidenciales de los parámetros, un procedimiento apropiado para variables continuas no normales (Preacher \& Hayes, 2008). Se optó por estimar un modelo de mediación con las puntuaciones de las escalas, en vez de un modelo de ecuaciones estructurales (SEM, por sus siglas en inglés) compuesto por variables latentes, debido a que: (a) el tamaño muestral con que se contaba no era óptimo para un modelo complejo con 82 ítems y 6 variables latentes (Bandalos, 2008); (b) a que los modelos SEM, a pesar de proporcionar valores menos sesgados, tienen mayor variabilidad muestral y menor potencia (Hayes, Montoya \& Rockwood, 2017; Ledgerwood \& Shrout, 2011) y (c) a que sería necesario estimar un modelo exploratorio de ecuaciones estructurales que requeriría de una muestra mayor al estimar todas las cargas cruzadas, ya que los modelos SEM basados en estructuras simples tienden a ofrecer parámetros sesgados con datos reales cuyos indicadores suelen ser impuros (Hsu, Skidmore, Li \& Thompson, 2014; Xiao, Liu, \& Hau, 2019).

El manejo y cribado de los datos, la imputación de los valores perdidos, los análisis descriptivos, la consistencia interna, las correlaciones de Pearson y las comparaciones por género se llevaron a cabo utilizando el programa SPSS versión 20. Para los análisis de mediación se utilizó el programa Mplus versión 7.4.

\section{Resultados}

En la Tabla 2 se muestran los estadísticos descriptivos para las escalas de los instrumentos aplicados. En el caso del EVI, la media obtenida corresponde aproximadamente a la segunda opción de respuesta en desacuerdo, mientras que para el ANS, la media estuvo cerca del punto intermedio $(2,50)$ de las opciones de respuesta en desacuerdo y de acuerdo. En el caso de la SRP, la media quedó por encima del punto intermedio de 3,50 para seis opciones puntuadas del 1 al 6. Para las CNI, la media, con cinco opciones puntuadas del 1 al 5, indica que los respondientes, en general, están de acuerdo o totalmente de acuerdo con que la infidelidad afecta de manera negativa a la relación de pareja. Por el contrario, la media para las CPI indica que están aproximadamente en desacuerdo con que la infidelidad tiene consecuencias positivas para la relación de pareja. Respecto a la infidelidad sexual, la media indica que en promedio los respondientes han cometido actos de infidelidad entre nunca y rara vez.

En lo que respecta a las diferencias de género en las puntuaciones de las escalas (Tabla 2), los resultados de la Prueba $T$ de Welch indicaron que solo había diferencias en las escalas de CPI e infidelidad sexual. En el caso de la escala de CPI, los hombres obtuvieron una puntuación media mayor a la de las mujeres. Similarmente, la media de los hombres en la escala de infidelidad sexual fue mayor que la de las mujeres. Estos resultados indican que los hombres cometen más actos de infidelidad sexual y que tienden a ver más consecuencias positivas de estos actos para la relación de pareja.

En la Tabla 3 se muestran en la diagonal los coeficientes de consistencia interna alfa de Cronbach para las escalas. Según los resultados obtenidos, las escalas de CPI y CNI alcanzaron un nivel aceptable de consistencia interna, las de ANS y EVI, un nivel bueno y las de SRP e infidelidad sexual, un nivel excelente. 
Tabla 3

Correlaciones entre las Variables Control y las Escalas

\begin{tabular}{lllllllllll}
\hline \multicolumn{1}{c}{ Variable } & 1 & 2 & 3 & 4 & 5 & 6 & 7 & 8 & 9 & 10 \\
\hline 1. Edad & 1,00 & & & & & & & & & \\
2. Sexo & 0,05 & 1,00 & & & & & & & & \\
3. Educación & $0,55^{* *}$ & $-0,11^{*}$ & 1,00 & & & & & & & \\
4. DurRec & $0,78^{* *}$ & $-0,09$ & $0,45^{* *}$ & 1,00 & & & & & & \\
5. EVI & $0,14^{* *}$ & $-0,02$ & $0,14^{* *}$ & 0,09 & $(0,84)$ & & & & & \\
6. ANS & $-0,09$ & $-0,07$ & $-0,08$ & $-0,05$ & $0,15^{* *}$ & $(0,84)$ & & & & \\
7. SRP & $-0,24^{* *}$ & $-0,05$ & $-0,21^{* *}$ & $-0,07$ & $-0,62^{* *}$ & $-0,13^{*}$ & $(0,95)$ & & & \\
8. CNI & $-0,22^{* *}$ & $-0,08$ & $-0,04$ & $-0,19^{* *}$ & $-0,10^{* *}$ & 0,03 & 0,09 & $(0,79)$ & & \\
9. CPI & $0,18^{* *}$ & $0,21^{* *}$ & $0,15^{* *}$ & $0,10^{*}$ & $0,19^{* *}$ & $0,13^{*}$ & $-0,27^{* * *}$ & $-0,39^{* *}$ & $(0,78)$ & \\
10. IS & 0,09 & $0,37^{* *}$ & 0,07 & $-0,00$ & $0,23^{* *}$ & $0,13^{* *}$ & $-0,30^{* *}$ & $-0,18^{* *}$ & $0,44^{* *}$ & $(0,98)$ \\
\hline
\end{tabular}

Nota. $N$ = 403; DurRec = Duración de la relación de pareja; EVI = Apego evitativo; ANS = Apego ansioso; SRP = Satisfacción con la relación de pareja; $\mathrm{CNI}=$ Consecuencias negativas de la infidelidad; $\mathrm{CPI}=$ Consecuencias positivas de la infidelidad; IS = Infidelidad sexual. La consistencia interna de las escalas según alfa de Cronbach aparece entre paréntesis en la diagonal. La variable sexo se codificó mujer $=0$, hombre $=1$. ${ }^{*} p<0,05 ;{ }^{* *} p<0,01$.

Los coeficientes de correlación de Pearson entre las escalas y las variables control se muestran también en la Tabla 3. Como se puede observar ahí, todas las correlaciones entre las escalas fueron estadísticamente significativas, excepto la correlación de las CNI con el ANS $(p=0,574)$ y con la SRP $(p=0,086)$. Tanto el EVI $(p<0,001)$ como el ANS $(p=0,011)$ se relacionaron de manera inversa con la SRP, pero la correlación con el EVI fue mucho más alta. También cabe destacar que todas las escalas correlacionaron con la infidelidad sexual, directamente en el caso del EVI $(p<0,001)$, el ANS $(p=0,008)$ y las CPI $(p<0,001)$ e inversamente con la SRP $(p<0,001)$ y las CNI $(p<0,001)$. Con respecto a las correlaciones entre las variables control y las escalas, se encontró que tener más edad estuvo asociado con tener un mayor EVI $(p=0,005)$ y CPI $(p<0,001)$ y una menor SRP $(p<0,001)$ y CNI $(p<0,001)$. En el caso del sexo, ser hombre estuvo asociado con mayor CPI $(p<0,001)$ e infidelidad sexual $(p<0,001)$. Adicionalmente, un mayor nivel educativo estuvo relacionado con más EVI $(p=0,006)$ y CPI $(p=0,003)$ y con menos SRP $(p<0,001)$. Finalmente, tener una relación de pareja por más tiempo se relacionó con más CPI $(p=0,045)$ y menos CNI $(p<0,001)$.

El diagrama de rutas correspondiente al modelo de mediación de la infidelidad se presenta en la Figura 1, incluyendo los pesos estandarizados de los efectos directos y la varianza explicada de las variables. Como se puede ver en el diagrama, la relación entre el apego adulto y la infidelidad sexual estaría mediada por la SRP y las percepciones de las CNI y CPI. Por simplicidad se omitieron del diagrama las variables control, las cuales se modelaron como variables exógenas que correlacionaban con el EVI y el ANS y que tenían efecto sobre las tres variables mediadoras y la infidelidad sexual (ver Tabla 4). Uno de los resultados notorios que se pueden observar en la Figura 1 es que los efectos directos del apego adulto (tanto del EVI como del ANS) a la infidelidad sexual dejan de ser significativos en el modelo de mediación. También se puede observar cómo los efectos directos del ANS a SRP y CNI no alcanzaron la significación estadística, al igual que el efecto directo de las CNI a la infidelidad sexual. Cabe destacar, además, que las variables del modelo lograron explicar un $33 \%$ de la varianza de la infidelidad sexual.

Los parámetros no estandarizados y estandarizados del modelo de mediación de la Figura 1 se muestran en la Tabla 4, así como sus intervalos confidenciales del 95\%. En el caso de la variable EVI, de los tres posibles efectos indirectos, dos resultaron significativos. El efecto indirecto estandarizado EVI $\rightarrow$ SRP $\rightarrow$ IS se podría categorizar como un efecto aproximadamente mediano, mientras que el efecto indirecto estandarizado EVI $\rightarrow$ CPI $\rightarrow$ IS podría considerarse como un efecto pequeño. Estos resultados indican que las variables de SRP y las CPI mediaron la relación entre el EVI y la infidelidad sexual. El restante efecto indirecto del EVI (EVI $\rightarrow$ CNI $\rightarrow$ IS) no fue significativo, indicando que CNI no medió la relación entre el EVI y la infidelidad sexual. Con respecto al ANS, se encontró que CPI medió su relación con la infidelidad sexual (ANS $\rightarrow$ CPI $\rightarrow$ IS), obteniéndose un efecto indirecto estandarizado que se podría considerar como pequeño. Los otros dos efectos indirectos (ANS $\rightarrow$ SRP $\rightarrow$ IS y ANS $\rightarrow$ CNI $\rightarrow$ IS) no resultaron significativos, indicando que SRP y CNI no mediaron la relación entre el ANS y la infidelidad sexual. 


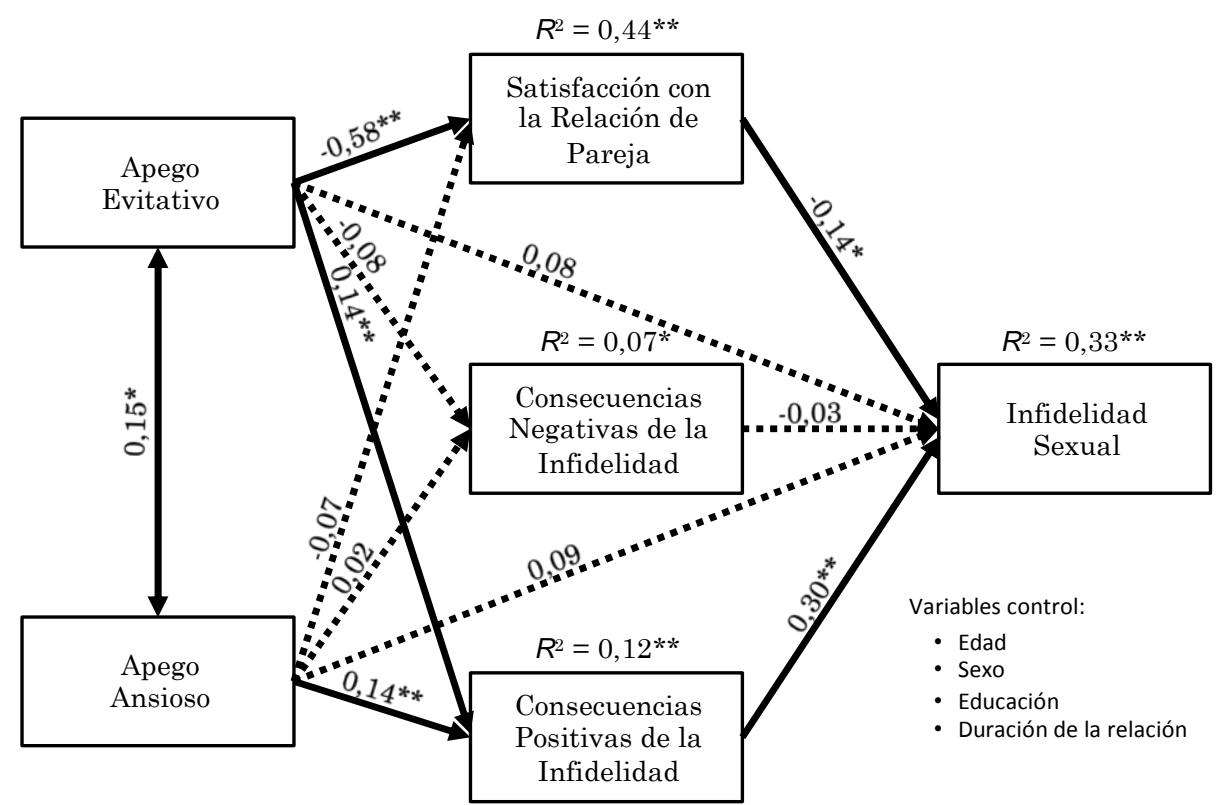

Figura 1. Modelo estandarizado de mediación de la infidelidad. $R^{2}=$ varianza explicada. Las flechas unidireccionales completas denotan regresiones significativas y las punteadas, regresiones no significativas. Las flechas bidireccionales completas denotan correlaciones significativas. Por simplicidad, se omiten del gráfico las variables control y las correlaciones entre los errores de las variables mediadoras. ${ }^{*} p<0,05,{ }^{* *} p<0,01$.

Tabla 4 Parámetros Estimados del Modelo de Mediación de la Infidelidad

\begin{tabular}{|c|c|c|c|c|}
\hline \multirow[b]{2}{*}{ Tipo de efecto/Efecto } & \multicolumn{2}{|c|}{ No estandarizados } & \multicolumn{2}{|c|}{ Estandarizados } \\
\hline & $\begin{array}{c}\text { Valor } \\
\text { estimado }\end{array}$ & $95 \%$ IC & $\begin{array}{l}\text { Valor } \\
\text { estimado }\end{array}$ & $95 \%$ IC \\
\hline \multicolumn{5}{|l|}{ Efectos de EVI a IS } \\
\hline Total & $0,325^{* *}$ & $0,170,0,483$ & $0,206^{* *}$ & $0,109,0,301$ \\
\hline Total indirecto & $0,202^{* *}$ & $0,078,0,333$ & $0,128^{* *}$ & $0,049, \quad 0,213$ \\
\hline \multicolumn{5}{|l|}{ Específicos indirectos } \\
\hline $\mathrm{EVI} \rightarrow \mathrm{SRP} \rightarrow \mathrm{IS}$ & $0,130^{*}$ & $0,010,0,255$ & $0,082^{*}$ & $0,006,0,165$ \\
\hline $\mathrm{EVI} \rightarrow \mathrm{CNI} \rightarrow \mathrm{IS}$ & 0,003 & $-0,012, \quad 0,021$ & 0,002 & $-0,007,0,014$ \\
\hline $\mathrm{EVI} \rightarrow \mathrm{CPI} \rightarrow \mathrm{IS}$ & $0,068^{* *}$ & $0,019,0,134$ & $0,043^{* *}$ & $0,012, \quad 0,083$ \\
\hline \multicolumn{5}{|l|}{ Directo } \\
\hline $\mathrm{EVI} \rightarrow \mathrm{IS}$ & 0,123 & $-0,041,0,289$ & 0,078 & $-0,026,0,178$ \\
\hline \multicolumn{5}{|l|}{ Efectos de ANS a IS } \\
\hline Total & $0,198^{*}$ & $0,039,0,360$ & $0,137^{*}$ & $0,027,0,246$ \\
\hline Total indirecto & $0,073^{* *}$ & $0,021,0,134$ & $0,051^{* *}$ & $0,014, \quad 0,092$ \\
\hline \multicolumn{5}{|l|}{ Específicos indirectos } \\
\hline $\mathrm{ANS} \rightarrow \mathrm{SRP} \rightarrow \mathrm{IS}$ & 0,014 & $-0,003,0,043$ & 0,010 & $-0,002,0,030$ \\
\hline $\mathrm{ANS} \rightarrow \mathrm{CNI} \rightarrow \mathrm{IS}$ & $-0,001$ & $-0,011,0,008$ & $-0,001$ & $-0,008,0,006$ \\
\hline $\mathrm{ANS} \rightarrow \mathrm{CPI} \rightarrow \mathrm{IS}$ & $0,060^{* *}$ & $0,014,0,120$ & $0,042^{* *}$ & $0,010,0,082$ \\
\hline \multicolumn{5}{|l|}{ Directo } \\
\hline $\mathrm{ANS} \rightarrow \mathrm{IS}$ & 0,125 & $-0,023,0,273$ & 0,087 & $-0,016,0,187$ \\
\hline \multicolumn{5}{|l|}{ Efectos directos restantes } \\
\hline $\mathrm{EVI} \rightarrow \mathrm{SRP}$ & $-1,338 * *$ & $-1,518,-1,146$ & $-0,579 * *$ & $-0,649,-0,498$ \\
\hline $\mathrm{ANS} \rightarrow \mathrm{SRP}$ & $-0,141$ & $-0,327,0,033$ & $-0,067$ & $-0.155,0,016$ \\
\hline
\end{tabular}


Tabla 4 (conclusión)

Parámetros Estimados del Modelo de Mediación de la Infidelidad

\begin{tabular}{|c|c|c|c|c|}
\hline \multirow[b]{2}{*}{ Tipo de efecto/Efecto } & \multicolumn{2}{|c|}{ No estandarizados } & \multicolumn{2}{|c|}{ Estandarizados } \\
\hline & $\begin{array}{l}\text { Valor } \\
\text { estimado }\end{array}$ & $95 \%$ IC & $\begin{array}{c}\text { Valor } \\
\text { estimado }\end{array}$ & $95 \%$ IC \\
\hline Edad $\rightarrow$ SRP & $-0,040 * *$ & $-0,059,-0,020$ & $-0,311^{* *}$ & $-0,456,-0,159$ \\
\hline Sexo $\rightarrow$ SRP & $-0,085$ & $-0,264,0,085$ & $-0,036$ & $-0,113,0,035$ \\
\hline Educación $\rightarrow$ SRP & $-0,079$ & $-0,189,0,024$ & $-0,080$ & $-0,189,0,024$ \\
\hline DurRel $\rightarrow$ SRP & $0,039^{* *}$ & $0,018,0,060$ & $0,248^{* *}$ & $0,110,0,386$ \\
\hline $\mathrm{EVI} \rightarrow \mathrm{CNI}$ & $-0,132$ & $-0,291,0,020$ & $-0,083$ & $-0.179,0,012$ \\
\hline $\mathrm{ANS} \rightarrow \mathrm{CNI}$ & 0,033 & $-0,111,0.175$ & 0,023 & $-0,077,0,119$ \\
\hline $\mathrm{Edad} \rightarrow \mathrm{CNI}$ & $-0,018^{*}$ & $-0,032,-0,005$ & $-0,208^{* *}$ & $-0,360,-0,052$ \\
\hline Sexo $\rightarrow$ CNI & $-0,100$ & $-0,261,0,056$ & $-0,061$ & $-0,162,0,034$ \\
\hline Educación $\rightarrow$ CNI & $0,078^{*}$ & $0,001,0,151$ & $0,114^{*}$ & $0,002,0,220$ \\
\hline DurRel $\rightarrow$ CNI & $-0,008$ & $-0,026,0,010$ & $-0,076$ & $-0,237,0,097$ \\
\hline $\mathrm{EVI} \rightarrow \mathrm{CPI}$ & $0,249 * *$ & $0,084,0,420$ & $0,142^{* *}$ & $0,048,0,235$ \\
\hline $\mathrm{ANS} \rightarrow \mathrm{CPI}$ & $0,220 * *$ & $0,059,0,380$ & $0,137 * *$ & $0,037,0,237$ \\
\hline Edad $\rightarrow$ CPI & 0,011 & $-0,004,0,027$ & 0,116 & $-0,044,0,276$ \\
\hline Sexo $\rightarrow$ CPI & $0,398^{* *}$ & $0,224,0,584$ & $0,222^{* *}$ & $0,127,0,319$ \\
\hline Educación $\rightarrow$ CPI & 0,083 & $-0,007,0,176$ & 0,110 & $-0,009, \quad 0,232$ \\
\hline DurRel $\rightarrow$ CPI & $-0,003$ & $-0,021,0,016$ & $-0,025$ & $-0,178,0,124$ \\
\hline $\mathrm{SRP} \rightarrow \mathrm{IS}$ & $-0,097^{*}$ & $-0,186,-0,008$ & $-0,142^{*}$ & $-0,276,-0,011$ \\
\hline $\mathrm{CNI} \rightarrow \mathrm{IS}$ & $-0,025$ & $-0,127,0,074$ & $-0,026$ & $-0,127,0,073$ \\
\hline $\mathrm{CPI} \rightarrow \mathrm{IS}$ & $0,274^{* *}$ & $0,161, \quad 0,389$ & $0,304^{* *}$ & $0,183,0,420$ \\
\hline Edad $\rightarrow$ IS & $-0,002$ & $-0,017,0,012$ & $-0,026$ & $-0,192,0,137$ \\
\hline Sexo $\rightarrow$ IS & $0,499 * *$ & $0,339, \quad 0,660$ & $0,310^{* *}$ & $0,211,0,407$ \\
\hline Educación $\rightarrow$ IS & 0,032 & $-0,042,0,109$ & 0,047 & $-0,063,0,158$ \\
\hline DurRel $\rightarrow$ IS & $-0,003$ & $-0,018, \quad 0,014$ & $-0,026$ & $-0,169,0,126$ \\
\hline
\end{tabular}

Nota . EVI = Apego evitativo; ANS = Apego ansioso; $\mathrm{SRP}=$ Satisfacción con la relación de pareja; $\mathrm{CNI}=$ Consecuencias negativas de la infidelidad; CPI = Consecuencias positivas de la infidelidad; IS = Infidelidad sexual; IC = Intervalo confidencial; DurRel = Duración de la relación de Pareja. La variable sexo se codificó mujer $=0$, hombre $=1$.

${ }^{*} p<0,05,{ }^{* *} p<0,01$.

\section{Discusión}

Hoy en día la infidelidad es frecuente en las relaciones de pareja (DeWall et al., 2011; Russell et al., 2013), impactando considerablemente en la calidad de vida de las personas involucradas en dicha relación, sus familias y la sociedad en general (Giraldo Hurtado \& Garcés, 2013; Rivera Aragón et al., 2011; Russell et al., 2013). Entre las variables más relevantes que se han relacionado con la infidelidad está el apego adulto, teniéndose que las personas con apego inseguro suelen ser más propensas a ser infieles, en especial aquellas con EVI (DeWall et al., 2011). Adicionalmente, numerosos estudios han vinculado la infidelidad a la insatisfacción en la relación de pareja y las actitudes positivas hacia estos comportamientos (DeWall et al., 2011; Norona et al., 2018; Romero-Palencia et al., 2008; Ziv et al., 2018). Bajo este contexto, el presente estudio buscó esclarecer la relación entre el apego adulto y la infidelidad sexual, considerando tres posibles mediadores: la SRP, las CPI de la infidelidad y las CNI de la infidelidad.

\section{Hallazgos Principales}

Los resultados apoyaron las hipótesis de que la infidelidad sexual se relacionaría directamente con el EVI, el ANS y las CPI e inversamente con la SRP y las CNI. Hallazgos similares fueron reportados por DeWall et al. (2011), quienes, a través de ocho estudios, encontraron que las personas con EVI mantenían actitudes más abiertas respecto de las relaciones de pareja y eran más frecuentemente infieles. Específicamente, las personas con EVI tienen dificultad para comprometerse y sentirse dependientes de su pareja. Tal dificultad podría ser percibida por las personas como baja intimidad dentro de la relación (Russell et al., 2013; Urrego Bentancourt, Gaitán Rodríguez \& Umbarila Forero, 2016), coincidiendo, además, con los resultados de Norona et al. (2018), quienes observaron que las personas que se aventuran 
fuera de su relación primaria pretenden llenar necesidades insatisfechas de independencia, interdependencia e intimidad. En esta línea, tanto el EVI como el ANS correlacionaron inversamente con la SRP, siendo la magnitud de dicha relación sustancialmente mayor para el EVI. Esto es congruente con los resultados metaanalíticos de Li y Chan (2012), los cuales mostraron que tanto la ansiedad como la evitación obstaculizaban la calidad de la relación y que, en comparación con el ANS, el EVI estaba más asociado con la satisfacción general en la relación de pareja.

Respecto del objetivo central del estudio, la evaluación de las posibles variables mediadoras de la relación entre el apego adulto y la infidelidad sexual, los resultados indican que: (a) la SRP media la relación entre el EVI y la infidelidad sexual (efecto aproximadamente mediano), pero no la relación entre el ANS y la infidelidad sexual; (b) las percepciones de las CPI median la relación entre ambos tipos de apego inseguro y la infidelidad sexual (efectos pequeños); (c) las percepciones de las CNI no median la relación entre los apegos inseguros y la infidelidad sexual y (d) los efectos directos del EVI y el ANS hacia la infidelidad sexual dejan de ser significativos al considerar el proceso de mediación completo. Estos hallazgos indican que el EVI, en congruencia con las expectativas del estudio, podría llevar a relaciones de pareja menos satisfactorias y a percepciones más positivas de las consecuencias de la infidelidad y ambas, a su vez, a mayor infidelidad. Esto concuerda con DeWall et al. (2011), quienes plantearon que la incomodidad crónica con la cercanía e intimidad de las personas con EVI tiene un efecto en sus actitudes hacia engañar a su pareja, en su nivel de compromiso y, por lo tanto, en cuánto se involucran en la infidelidad. Este comportamiento socava el bienestar de las relaciones, provocando una baja SRP y llevando en algunos casos a la disolución de la misma (Jeanfreau, Jurich \& Mong, 2014; Russell et al., 2013).

En el caso del ANS, y contrario a las hipótesis planteadas, la SRP no ejerce un rol mediador respecto de la infidelidad sexual. Más aún, en el modelo de mediación que incluye ambos tipos de apego (y que, por lo tanto, permite separar estadísticamente el efecto de cada uno), el ANS no ejerce un efecto sobre la SRP. Este resultado sugiere que la relación entre el ANS y la SRP podría estar explicada por aquellas características que comparte con el EVI (ambos inseguros) y no por aspectos específicos del mismo. Estos resultados concuerdan parcialmente con el metaanálisis de Li y Chan (2012), quienes encontraron que el ANS estaba menos relacionado con la SRP que el EVI. Con respecto al rol mediador de las percepciones de las CPI sobre el ANS y la infidelidad sexual, los resultados encontrados apoyan la hipótesis de mediación planteada y son contrarios a los obtenidos por DeWall et al. (2011), quienes no hallaron un efecto de mediación de las actitudes positivas hacia la infidelidad para este tipo de apego. Es posible que la necesidad que tienen las personas con ANS de lograr una mayor intimidad haga que tengan actitudes más positivas hacia la infidelidad, constituyendo el sexo extradiádico una forma de satisfacer esta necesidad (Russell et al., 2013). Por otro lado, las percepciones de las CNI no tuvieron un efecto mediador para ninguno de los apegos inseguros, contrario a las expectativas del estudio, indicando que la infidelidad sexual está impactada principalmente por los efectos de acción que ejercen las actitudes positivas, a diferencia de los efectos de inhibición que pudiesen ejercer las actitudes negativas hacia la infidelidad. De hecho, en el modelo de mediación las percepciones de las CNI no ejercen un efecto sobre la infidelidad sexual, indicando que, luego de considerar los otros dos mediadores, esta variable no es relevante respecto de la infidelidad sexual.

Finalmente, los resultados del presente estudio verifican que existen diferencias de género en la infidelidad sexual, teniéndose que los hombres cometen más actos de infidelidad sexual que las mujeres y ven más consecuencias positivas de estos actos para la relación de pareja. Resultados similares han sido reportados en numerosas investigaciones (e.g., Barta \& Kiene, 2005; Frederick \& Fales, 2016; Mark et al., 2011), comenzando por los estudios pioneros de Kinsey, Pomeroy, Martin y Gebhard (1953). En este sentido, Allen et al. (2005) plantean que los hombres tienen mayor probabilidad de participar en una infidelidad que las mujeres, aspectos que se asocian a los roles de género, a los procesos de socialización, a las creencias sociales sobre lo propio para cada sexo y a las diferencias culturales (Buunk \& Dijkstra, 2015).

\section{Limitaciones del Estudio y Direcciones para el Futuro}

Los hallazgos del presente estudio deben considerase en el contexto de sus limitaciones. Por un lado, debido al tamaño de la muestra recolectada, no se pudo evaluar el posible rol moderador del género y la orientación sexual en la mediación de la relación entre el apego adulto y la infidelidad sexual, algo que se recomienda hacer en estudios futuros. Asimismo, se recolectó una muestra de estudiantes universitarios, por lo que cualquier generalización de los hallazgos aquí encontrados debería hacerse con cautela. No 
obstante, es preciso destacar que parte de los resultados obtenidos son congruentes con la literatura internacional, como, por ejemplo, la mayor relación del EVI, en comparación con el ANS, con la SRP y la infidelidad (DeWall et al., 2011; Li \& Chan, 2012), lo que apoya la robustez de los mismos. Adicionalmente, se estimó un modelo de mediación basado en las puntuaciones de las escalas, en vez de un modelo SEM con variables latentes. Debido a esto, es esperable que los pesos de regresión estimados tengan sesgo (posiblemente estén disminuidos), debido a que no hay corrección por falta de fiabilidad de las puntuaciones, entre otras cosas. A pesar de esto, en el estudio actual este problema estaría mitigado por el hecho de que las puntuaciones en las escalas en general presentaron fiabilidades elevadas (Ledgerwood \& Shrout, 2011). Por otro lado, modelos de mediación alternativos al propuesto en el presente estudio no pueden ser descartados, debido al carácter transversal del estudio y a la estimación de un modelo saturado.

Se conocen pocos estudios que hayan reportado los efectos interactivos de los estilos de apego propio y el de la pareja para predecir la infidelidad y su relación con la SRP. La manera en la cual el tipo de apego propio predice la infidelidad puede estar relacionada con el tipo de apego de la pareja y el género, por lo que se propone que futuras investigaciones se interesen en conocer el efecto de la interacción de los apegos en la relación de pareja sobre estas variables.

\section{Implicaciones Prácticas}

Los hallazgos de esta investigación pueden proporcionar a los terapeutas, familiares e individuales, una mejor comprensión de los procesos que pueden llevar a las personas a ser infieles. Dado que las personas con apego inseguro son más vulnerables a experimentar niveles más bajos de SRP, parte de los objetivos terapéuticos pueden centrarse en el trabajo de las dinámicas negativas propias de dichos estilos de apego (e.g., sensibilidad hacia el rechazo, hipervigilancia por el temor a la pérdida, incomodidad con la cercanía) que afectan de manera negativa a la calidad de la relación de pareja. Adicionalmente, profundizar sobre cómo los estilos de apego, en específico el EVI, pueden llevar a tener actitudes más positivas hacia la infidelidad, puede abrir vías terapéuticas para abordar la problemática de la infidelidad y lograr cambios positivos en las personas y las relaciones.

\section{Referencias}

Afifi, W. A., Falato, W. L. \& Weiner, J. L. (2001). Identity concerns following a severe relational transgression: The role of discovery method for the relational outcomes of infidelity. Journal of Social and Personal Relationships, 18, 291-308. https://doi.org/10.1177/0265407501182007

Allen, E. S., Atkins, D. C., Baucom, D. H., Snyder, D. K., Gordon, K. C. \& Glass, S. P. (2005). Intrapersonal, interpersonal, and contextual factors in engaging in and responding to extramarital involvement. Clinical Psychology: Science and Practice, 12, 101130. https://doi.org/10.1093/clipsy.bpi014

Allen, E. S., Rhoades, G. K., Stanley, S. M., Loew, B. \& Markman, H. J. (2012). The effects of marriage education for army couples with a history of infidelity. Journal of Family Psychology, 26, 26-35. https://doi.org/10.1037/a0026742

Alonso-Arbiol, I., Balluerka, N. \& Shaver, P. R. (2007). A Spanish version of the Experiences in Close Relationships (ECR) Adult Attachment Questionnaire. Personal Relationships, 14, 45-63. https://doi.org/10.1111/j.1475-6811.2006.00141.x

Bandalos, D. L. (2008). Is parceling really necessary? A comparison of results from item parceling and categorical variable methodology. Structural Equation Modeling, 15, 211-240. https://doi.org/10.1080/10705510801922340

Barta, W. D. \& Kiene, S. M. (2005). Motivations for infidelity in heterosexual dating couples: The roles of gender, personality differences, and sociosexual orientation. Journal of Social and Personal Relationships, 22, 339-360. https://doi.org/10.1177/0265407505052440

Behling, O. \& Law, K. S. (2000). Translating questionnaires and other research instruments: Problems and solutions. Thousand Oaks, CA: SAGE. https://doi.org/10.4135/9781412986373

Boekhout, B. A., Hendrick, S. S. \& Hendrick, C. (2003). Exploring infidelity: Developing the Relationship Issues Scale. Journal of Loss and Trauma, 8, 283-306. https://doi.org/10.1080/15325020305882

Bowlby, J. (1969/1998). El apego y la pérdida. Volumen I: El apego (M. Valcarce, Trad.; Título original: Attachment and loss. I. Attachment). Barcelona, España: Paidós.

Brennan, K. A., Clark, C. L. \& Shaver, P. R. (1998). Self- report measurement of adult attachment: An integrative overview. En J. A. Simpson \& W. S. Rholes (Eds.), Attachment theory and close relationships (pp. 46-76). New York, NY: Guilford Press.

Butzer, B. \& Campbell, L. (2008). Adult attachment, sexual satisfaction, and relationship satisfaction: A study of married couples. Personal Relationships, 15, 141-154. https://doi.org/10.1111/j.1475-6811.2007.00189.x

Buunk, A. P. \& Dijkstra, P. (2015). Rival characteristics that provoke jealousy: A study in Iraqi Kurdistan. Evolutionary Behavioral Sciences, 9, 116-127. https://doi.org/10.1037/ebs0000030

Campbell, L., Simpson, J. A., Boldry, J. \& Kashy, D. A. (2005). Perceptions of conflict and support in romantic relationships: The role of attachment anxiety. Journal of Personality and Social Psychology, 88, 510-531. https://doi.org/10.1037/0022-3514.88.3.510

Cohen, J. (1992). A power primer. Psychological Bulletin, 112, 155-159. https://doi.org/10.1037//0033-2909.112.1.155

Cole, J. (1997/2002). Dificultades y conflictos de pareja (F. Ruiz, Trad.; Título original: Crunch points for couples). Barcelona, España: Hispano Europea. 
Davis, D., Shaver, P. R. \& Vernon, M. L. (2003). Physical, emotional, and behavioral reactions to breaking up: The roles of gender, age, emotional involvement, and attachment style. Personality and Social Psychology Bulletin, 29, 871-884. https://doi.org/10.1177/0146167203029007006

Delacre, M., Lakens, D. \& Leys, C. (2017). Why psychologists should by default use Welch's $t$-test instead of Student's $t$-test. International Review of Social Psychology, 30, 92-101. https://doi.org/10.5334/irsp.82

DeSimone, J. A., Harms, P. D. \& DeSimone, A. J. (2015). Best practice recommendations for data screening. Journal of Organizational Behavior, 36, 171-181. https://doi.org/10.1002/job.1962

DeWall, C. N., Lambert, N. M., Slotter, E. B., Pond Jr., R. S., Deckman, T., Finkel, E. J. ... Fincham, F. D. (2011). So far away from one's partner, yet so close to romantic alternatives: Avoidant attachment, interest in alternatives, and infidelity. Journal of Personality and Social Psychology, 101, 1302-1316. https://doi.org/10.1037/a0025497

Elsner, P., Montero, M. L., Reyes, C. \& Zegers, B. (2000). La familia: una aventura. Santiago, Chile: Ediciones Universidad Católica de Chile.

Fincham, F. D. \& May, R. W. (2017). Infidelity in romantic relationships. Current Opinion in Psychology, 13, 70-74. https://doi.org/10.1016/j.copsyc.2016.03.008

Fisher, H. E. (1992/2007). Anatomía del amor: historia natural de la monogamia, el adulterio y el divorcio (2a ed.; A. Plante, Trad.; Título original: Anatomy of love: The natural history of monogamy, adultery and divorce). Barcelona, España: Anagrama.

Fox-Wasylyshyn, S. M. \& El-Masri, M. M. (2005). Handling missing data in self-report measures. Research in Nursing \& Health, 28, 488-495. https://doi.org/10.1002/nur.20100

Fraley, R. C. \& Shaver, P. R. (2000). Adult romantic attachment: Theoretical developments, emerging controversies, and unanswered questions. Review of General Psychology, 4, 132-154. https://doi.org/10.1037/1089-2680.4.2.132

Frederick, D. A. \& Fales, M. R. (2016). Upset over sexual versus emotional infidelity among gay, lesbian, bisexual, and heterosexual adults. Archives of Sexual Behavior, 45, 175-191. https://doi.org/10.1007/s10508-014-0409-9

Fritz, M. S. \& MacKinnon, D. P. (2007). Required sample size to detect the mediated effect. Psychological Science, 18, $233-239$. https://doi.org/10.1111/j.1467-9280.2007.01882.x

Funk, J. L. \& Rogge, R. D. (2007). Testing the ruler with item response theory: Increasing precision of measurement for relationship satisfaction with the Couples Satisfaction Index. Journal of Family Psychology, 21, 572-583. https://doi.org/10.1037/0893-3200.21.4.572

George, D. \& Mallery, P. (2003). SPSS for Windows step by step: A simple guide and reference 11.0 update (4a ed.). Boston, MA: Allyn \& Bacon.

Giraldo Hurtado, C. M. \& Garcés, M. H. (2013). Emociones asociadas al descubrimiento de la infidelidad: de la felicidad a la infelicidad. Revista de la Facultad de Trabajo Social, 29, 143-157. Extraído de https://revistas.upb.edu.co/index.php/trabajosocial/article/view/2331/2075

Guitar, A. E., Geher, G., Kruger, D. J., Garcia, J. R., Fisher, M. L. \& Fitzgerald, C. J. (2017). Defining and distinguishing sexual and emotional infidelity. Current Psychology, 36, 434-446. https://doi.org/10.1007/s12144-016-9432-4

Hair Jr., J. F., Black, W. C., Babin, B. J. \& Anderson, R. E. (2010). Multivariate data analysis: A global perspective (7ª ed.). Upper Saddle River, NJ: Pearson Education.

Hall, J. H. \& Fincham, F. D. (2009). Psychological distress: Precursor or consequence of dating infidelity? Personality and Social Psychology Bulletin, 35, 143-159. https://doi.org/10.1177/0146167208327189

Hatfield, E. \& Rapson, R. L. (2006). Love and sexual health. En J. Kuriansky, M. S. Tepper \& A. F. Owens (Eds.), Sex, love, and psychology: Sexual health (pp. 93-97). New York, NY: Praeger.

Hayes, A. F., Montoya, A. K. \& Rockwood, N. J. (2017). The analysis of mechanisms and their contingencies: PROCESS versus structural equation modeling. Australasian Marketing Journal, 25, 76-81. https://doi.org/10.1016/j.ausmj.2017.02.001

Hayes, A. F. \& Scharkow, M. (2013). The relative trustworthiness of inferential tests of the indirect effect in statistical mediation analysis: Does method really matter? Psychological Science, 24, 1918-1927. https://doi.org/10.1177/0956797613480187

Hazan, C. \& Shaver, P. (1987). Romantic love conceptualized as an attachment process. Journal of Personality and Social Psychology, 52, 511-524. https://doi.org/10.1037/0022-3514.52.3.511

Hazan, C. \& Shaver, P. R. (1994). Attachment as an organizational framework for research on close relationships. Psychological Inquiry, 5, 1-22. https://doi.org/10.1207/s15327965pli0501_1

Hsu, H. -Y., Skidmore, S. T., Li, Y. \& Thompson, B. (2014). Forced zero cross-loading misspecifications in measurement component of structural equation models: Beware of even "small" misspecifications. Methodology, 10, 138-152. https://doi.org/10.1027/1614-2241/a000084

Icard, L. D., Jemmott III, J. B., Teitelman, A., O'Leary, A. \& Heeren, G. A. (2014). Mediation effects of problem drinking and marijuana use on HIV sexual risk behaviors among childhood sexually abused South African heterosexual men. Child Abuse \& Neglect, 38, 234-242. https://doi.org/10.1016/j.chiabu.2013.08.002

Jeanfreau, M. M., Jurich, A. P. \& Mong, M. D. (2014). Risk factors associated with women's marital infidelity. Contemporary Family Therapy, 36, 327-332. https://doi.org/10.1007/s10591-014-9309-3

Kinsey, A. C., Pomeroy, W. B., Martin, C. E. \& Gebhard, P. H. (1953). Sexual behavior in the human female. Philadelphia, PA: Saunders.

Kobak, R. D. \& Hazan, C. (1991). Attachment in marriage: Effects of security and accuracy of working models. Journal of Personality and Social Psychology, 60, 861-869. https://doi.org/10.1037/0022-3514.60.6.861

Ledgerwood, A. \& Shrout, P. E. (2011). The trade-off between accuracy and precision in latent variable models of mediation processes. Journal of Personality and Social Psychology, 101, 1174-1188. https://doi.org/10.1037/a0024776

Lewandowski, G. W. \& Ackerman, R. A. (2006). Something's missing: Need fulfillment and self-expansion as predictors of susceptibility to infidelity. The Journal of Social Psychology, 146, 389-403. https://doi.org/10.3200/SOCP.146.4.389-403

Li, T. \& Chan, D. K. -S. (2012). How anxious and avoidant attachment affect romantic relationship quality differently: A meta-analytic review. European Journal of Social Psychology, 42, 406-419. https://doi.org/10.1002/ejsp.1842

Logue, E. (2013). Extending the prediction of infidelity using a five-factor model (Tesis de Doctorado, Texas Tech University, Lubbock, TX, Estados Unidos). Extraído de http://hdl.handle.net/2346/58567

López Beltrán, M. I., Pérez Barberá, M. A., Piquer Barrachina, M. B. \& Ballester-Arnal, R. (2017). Motivos y reacciones ante la infidelidad en población universitaria. Àgora De Salut, 4, 215-222. https://doi.org/10.6035/AgoraSalut.2017.4.22

Loubat, M., Ponce, P. \& Salas, P. (2007). Estilo de apego en mujeres y su relación con el fenómeno del maltrato conyugal. Terapia Psicológica, 25, 113-122. https://doi.org/10.4067/S0718-48082007000200002

Maniaci, M. R. \& Rogge, R. D. (2014). Caring about carelessness: Participant inattention and its effects on research. Journal of Research in Personality, 48, 61-83. https://doi.org/10.1016/j.jpp.2013.09.008

Mark, K. P., Janssen, E. \& Milhausen, R. R. (2011). Infidelity in heterosexual couples: Demographic, interpersonal, and personalityrelated predictors of extradyadic sex. Archives of Sexual Behavior, 40, 971-982. https://doi.org/10.1007/s10508-011-9771-z 
Martins, A., Pereira, M., Andrade, R., Dattilio, F. M., Narciso, I. \& Canavarro, M. C. (2016). Infidelity in dating relationships: Genderspecific correlates of face-to-face and online extradyadic involvement. Archives of Sexual Behavior, 45, 193-205. https://doi.org/10.1007/s10508-015-0576-3

McDaniel, B. T., Drouin, M. \& Cravens, J. D. (2017). Do you have anything to hide? Infidelity-related behaviors on social media sites and marital satisfaction. Computers in Human Behavior, 66, 88-95. https://doi.org/10.1016/j.chb.2016.09.031

Meade, A. W. \& Craig, S. B. (2012). Identifying careless responses in survey data. Psychological Methods, 17, 437-455. https://doi.org/10.1037/a0028085

Medina, C. J., Rivera, L. Y. \& Aguasvivas, J. A. (2016). El apego adulto y la calidad percibida de las relaciones de pareja: evidencias a partir de una población adulta joven. Salud \& Sociedad, 7, 306-318. https://doi.org/10.22199/S07187475.2016.0003.00005

Mikulincer, M. \& Shaver, P. R. (2003). The attachment behavioral system in adulthood: Activation, psychodynamics, and interpersonal processes. Advances in Experimental Social Psychology, 35, 56-152. https://doi.org/10.1016/s0065-2601(03)01002-5

Mikulincer, M. \& Shaver, P. R. (2007) Boosting attachment security to promote mental health, prosocial values, and inter-group tolerance. Psychological Inquiry, 18, 139-156. https://doi.org/10.1080/10478400701512646

Norona, J. C., Olmstead, S. B. \& Welsh, D. P. (2018). Betrayals in emerging adulthood: A developmental perspective of infidelity. The Journal of Sex Research, 55, 84-98. https://doi.org/10.1080/00224499.2017.1342757

Parker, M. L. \& Campbell, K. (2017). Infidelity and attachment: The moderating role of race/ethnicity. Contemporary Family Therapy, 39, 172-183. https://doi.org/10.1007/s10591-017-9415-0

Preacher, K. J. \& Hayes, A. F. (2008). Asymptotic and resampling strategies for assessing and comparing indirect effects in multiple mediator models. Behavior Research Methods, 40, 879-891. https://doi.org/10.3758/BRM.40.3.879

Previti, D. \& Amato, P. R. (2004). Is infidelity a cause or a consequence of poor marital quality? Journal of Social and Personal Relationships, 21, 217-230. https://doi.org/10.1177/0265407504041384

Rasch, D., Kubinger, K. D. \& Moder, K. (2011). The two-sample $t$ test: Pre-testing its assumptions does not pay off. Statistical Papers, 52, 219-231. https://doi.org/10.1007/s00362-009-0224-x

Revilla, M. A., Saris, W. E. \& Krosnick, J. A. (2014). Choosing the number of categories in agree-disagree scales. Sociological Methods \& Research, 43, 73-97. https://doi.org/10.1177/0049124113509605

Rivera Aragón, S., Díaz Loving, R., Villanueva Orozco, G. B. T. \& Montero Santamaria, N. (2011). El conflicto como un predictor de la infidelidad. Acta de Investigación Psicológica, 1, 298-315. Extraído de http://www.scielo.org.mx/pdf/aip/v1n2/v1n2a7.pdf

Romero-Palencia, A., Cruz del Castillo, C. \& Díaz-Loving, R. (2008). Propuesta de un modelo bio-psico-sociocultural de infidelidad sexual y emocional en hombres y mujeres. Psicología Iberoamericana, 16(2), 14-21. Extraído de https://www.redalyc.org/pdf/1339/133920328003.pdf

Romero Palencia, A., Rivera Aragón, S. \& Díaz Loving, R. (2007). Desarrollo del Inventario Multidimensional de Infidelidad (IMIN). Revista Iberoamericana de Diagnóstico y Evaluación Psicológica, 1(23), 121-147. Extraído de https://www.redalyc.org/pdf/4596/459645446008.pdf

Ruiz, F. J. \& Odriozola-González, P. (2016). The role of psychological inflexibility in Beck's cognitive model of depression in a sample of undergraduates. Anales de Psicología, 32, 441-447. https://doi.org/10.6018/analesps.32.2.214551

Russell, V. M., Baker, L. R. \& McNulty, J. K. (2013). Attachment insecurity and infidelity in marriage: Do studies of dating relationships really inform us about marriage? Journal of Family Psychology, 27, 242-251. https://doi.org/10.1037/a0032118

Schmider, E., Ziegler, M., Danay, E., Beyer, L. \& Bühner, M. (2010). Is it really robust? Reinvestigating the robustness of ANOVA against violations of the normal distribution assumption. Methodology, 6, 147-151. https://doi.org/10.1027/1614-2241/a000016

Treas, J. \& Giesen, D. (2000). Sexual infidelity among married and cohabiting Americans. Journal of Marriage and Family, 62, 48-60. https://doi.org/10.1111/j.1741-3737.2000.00048.x

Urrego Bentancourt, Y., Gaitán Rodríguez, N. A. \& Umbarila Forero, D. A. (2016). Relación entre el tipo de apego y la conducta de infidelidad en adultos jóvenes. Revista de Psicología de la Pontificia Universidad Católica Argentina, 12(24), 41-54. Extraído de http://erevistas.uca.edu.ar/index.php/RPSI/article/view/413

Weijters, B., Cabooter, E. \& Schillewaert, N. (2010). The effect of rating scale format on response styles: The number of response categories and response category labels. International Journal of Research in Marketing, 27, $236-247$. https://doi.org/10.1016/j.ijresmar.2010.02.004

Weiser, D. A., Lalasz, C. B., Weigel, D. J. \& Evans, W. P. (2014). A prototype analysis of infidelity. Personal Relationships, 21, 655675. https://doi.org/10.1111/pere.12056

Widaman, K. F. (2006). Missing data: What to do with or without them. Monographs of the Society for Research in Child Development, 71(3), 42-64. Extraído de https://srcd.onlinelibrary.wiley.com/doi/epdf/10.1111/j.1540-5834.2006.00404.x

Xiao, Y., Liu, H. \& Hau, K. -T. (2019). A comparison of CFA, ESEM, and BSEM in test structure analysis. Structural Equation Modeling. Anticipo en línea de la publicación. https://doi.org/10.1080/10705511.2018.1562928. Extraído de https://www.tandfonline.com/doi/full/10.1080/10705511.2018.1562928

Zare, B. (2011). Review of studies on infidelity. En 3rd International Conference on Advanced Management Science, 19, 182-186. Kuala Lumpur, Malasia: Editor. Extraído de http://www.ipedr.com/vol19/34-ICAMS2011-A10054.pdf

Ziv, I., Lubin, O. B. -H. \& Asher, S. (2018). "I swear I will never betray you": Factors reported by spouses as helping them resist extramarital sex in relation to gender, marriage length, and religiosity. The Journal of Sex Research, 55, $236-251$. https://doi.org/10.1080/00224499.2017.1347602

Fecha de recepción: Diciembre de 2018.

Fecha de aceptación: Junio de 2019. 